Mathematical Sciences and Applications

E-NOTES

MATHEMATICAL

SCIENCES

AND APPLICATIONS

E-NOTES

https://doi.org/10.36753/mathenot.614732

9 (1) 28-35 (2021) - Research Article

ISSN: $2147-6268$

(C)MSAEN

\title{
Bernstein Operator Approach for Solving Linear Differential Equations
}

\author{
Neşe İşler Acar
}

\begin{abstract}
In this study, an alternative numerical method having regard to the Bernstein operator is generated for approximate solutions of linear differential equations in the most general form under the initial and boundary conditions. Some applications are also revealed to show how the procedure can be performed for the problems.
\end{abstract}

Keywords: Bernstein polynomials; Linear differential equations; Operator numerical method

AMS Subject Classification (2020): Primary: 34B05; Secondary: 65L05; 65L10; 65L80.

\section{Introduction}

Sergei Natanovich Bernstein have produced the Bernstein polynomials verified the Weierstrass Theorem [8] in 1912. Then, these polynomials have leaded the constructive function theory and have become center of the applied analysis. Considering the Bernstein polynomials as a sequence of linear positive operators, these polynomials hold the Korovkin Theorem [23]. For this reason, the Bernstein operator and their derivatives converge to a continuous function and their derivatives on a closed and bounded interval [24].

In ages, the generated numerical methods have been investigated by considering the Bernstein polynomials. Numerical examples of these polynomials have been come on differential equations [7, 10, 18, 27,36], integral equations [5, 35], integro-differential equations [6], partial differential equations [4, 39], fractional order equations [38] from past to present.

In recent years, there are several searches about reformed numerical methods based on the Berstein polynomials. Only in four years, collocation method that is one of the numerical method has been developed for numerical solution of many types of equations like integral equations [17], integro-differential equations [11,37] and differential equations [25]. The Bernstein polynomials have also been modified the Adomian decomposition [31] and Laplace decomposition method [33] for solving linear and nonlinear differential equations. The spectral Petrov-Galerkin method [20] has been applied for numerical solution of fractional partial differential equations that are one of the most popular and remarkable equations in the science world. Discretization method [13] that is other numerical method has been applied to the Volterra-Fredholm integral equations by utilizing the Bernstein polynomials. Improved variational iteration method [30] has been used for approximate solution of partial differential equations. 
Moreover, some operational matrix forms of the Bernstein polynomials have been generated for numerical methods. For example, operational matrix of shifted orthonormal Bernstein polynomials has been used for the numerical solution of pantograph equations [21], computational methods based on operational matrix of differentiation and integration via the Berntein polynomials has been presented for solving differential equations[3, 22, 28, 29, 39], Volterra integral equations [26] and fractional order differential equations [34].

However, in point of fact, numerical methods have not examined by taking the Bernstein operator instead of the Bernstein polynomials for numerical solutions of these types of equations. In this direction, our aim is to converge the solution of $m$ th-order linear differential equation

$$
\sum_{k=0}^{m} a_{k}(x) y^{(k)}(x)=g(x), a \leq x \leq b
$$

under the mixed conditions

$$
\sum_{k=0}^{m-1} \sum_{j=0}^{l} \alpha_{i j}^{k} y^{(k)}\left(c_{j}\right)=\beta_{i} ; i=0,1, \ldots, m-1, a \leq c_{j} \leq b .
$$

Here the solution, coefficient and source functions assume that have Bernstein operator defined on the interval $[a, b]$ as follows:

$$
\begin{aligned}
y^{(k)}(x) & \cong B_{n}^{(k)}(y ; x)=\sum_{i=0}^{n} y\left(a+\frac{i(b-a)}{n}\right) p_{i, n}^{(k)}(x), \\
g(x) & \simeq B_{n}(g, x)=\sum_{i=0}^{n} g\left(a+\frac{i(b-a)}{n}\right) p_{i, n}(x), \\
a_{k}(x) & \cong B_{n}\left(a_{k} ; x\right)=\sum_{i=0}^{n} a_{k}\left(a+\frac{i(b-a)}{n}\right) p_{i, n}(x) .
\end{aligned}
$$

Here $a_{k}(x), g(x) \in C[a, b]$, and $y(x)$ is unknown function. Moreover, $p_{i, n}(x)$ called the generalized Bernstein polynomials [14].

Theorem 1.1. The definite integral of the Bernstein basis polynomials on the interval $[a, b]$ has the following equality [11]

$$
\int_{a}^{b} p_{i, n}(x) d x=\frac{b-a}{n+1} ; 0 \leq i \leq n .
$$

Theorem 1.2. There is a relation between generalized Bernstein basis polynomials matrix and their derivatives in the form

$$
\mathbf{P}^{(k)}(x)=\mathbf{P}(x) \mathbf{N}^{k} ; k=0,1, \ldots, m .
$$

Here $\mathbf{P}(x)=\left[p_{i, n}(x)\right], \mathbf{P}^{k}(x)=\left[p_{i, n}^{(k)}(x)\right]$ are $1 \times(n+1)$ matrices, $\mathbf{N}=\left(d_{i j}\right)$ is $(n+1) \times(n+1)$ matrix such that the elements of $\mathbf{N}$ are defined by

$$
d_{i j}=\frac{1}{b-a}\left\{\begin{array}{ccl}
n-i & ; & \text { if } j=i+1 \\
2 i-n & ; & \text { if } j=i \\
-i & ; & \text { if } j=i-1 \\
0 & ; & \text { otherwise }
\end{array}\right.
$$

for $i, j=0,1, \ldots, n$ and $\mathbf{N}^{0}=\mathbf{I}$ is identity matrix [1].

The historical development of the Bernstein polynomials, derivated numerical methods by way of these polynomials, the definition of the differential equation, some main theorems and fundamental matrix relation required for the mentioned work are given in Section 1 . The new method is presented, how it is found out by using the Bernstein operator approach, in Section 2. The proposed method is shown how is applied to the differential equations by tables, and some errors used in the problems are defined in Section 3. In this part, four examples are also considered to see convergency of the method, and the numerical results of the proposed method and other methods are compared in the last example. Finally, in Section 4, advantages and disadvantages of the presented method are commented, and given some ideas about future studies. 


\section{Method of Solution}

Theorem 2.1. Let $y, a_{k}$ and $g$ functions have the Bernstein operator approach as given in (1.3), then the differential equation (1.1) holds the following matrix equation:

$$
\sum_{k=0}^{m} \mathcal{A}_{k} \mathcal{I} \mathbf{N}^{k} \mathcal{Y}=\mathcal{G}
$$

Here $\mathcal{Y}, \mathcal{A}_{k}$ and $\mathcal{G}$ are $(n+1) \times 1$ matrices and $I$ is $1 \times(n+1)$ matrix. Besides, the elements of these matrices are expressed as

$$
\begin{aligned}
\mathcal{Y} & =\left[\begin{array}{llll}
y(a) & y\left(a+\frac{b-a}{n}\right) & \ldots & y(b)
\end{array}\right]^{T}, \\
\mathcal{A}_{k} & =\left[\begin{array}{llll}
a_{k}(a) & a_{k}\left(a+\frac{b-a}{n}\right) & \ldots & a_{k}(b)
\end{array}\right]^{T}, \\
\mathcal{G} & =\left[\begin{array}{lllll}
g(a) & g\left(a+\frac{b-a}{n}\right) & \ldots & g(b)
\end{array}\right]^{T}, \\
\mathcal{I} & =\frac{1}{n+1}\left[\begin{array}{lllll}
1 & 1 & 1 & \ldots & 1
\end{array}\right] .
\end{aligned}
$$

Proof. Considering Bernstein operator approach (1.3) and Theorem 1.2, matrix forms of the $y, a_{k}$ and $g$ functions can be denoted as follows:

$$
\begin{aligned}
& y(x) \simeq \mathbf{P}(x) \mathcal{Y}, y^{(k)}(x) \simeq \mathbf{P}^{(k)}(x) \mathcal{Y}=\mathbf{P}(x) \mathbf{N}^{k} \mathcal{Y}, \\
& g(x) \simeq \mathbf{P}(x) \mathcal{G}, a_{k}(x) \simeq \mathbf{P}(x) \mathcal{A}_{k} .
\end{aligned}
$$

Substituting expressions (2.1) and (2.2) into equation (1.1), we obtain a linear algebraic equation

$$
\sum_{k=0}^{m} \mathbf{P}(x) \mathcal{A}_{k} \mathbf{P}(x) \mathbf{N}^{k} \mathcal{Y}=\mathbf{P}(x) \mathcal{G}
$$

and making some required simplifications, the equation can be rewritten as

$$
\sum_{k=0}^{m} \mathcal{A}_{k} \mathbf{P}(x) \mathbf{N}^{k} \mathcal{Y}=\mathcal{G}
$$

We integrate both sides of equation (2.3) on the interval $[a, b]$, then we have the following relations

$$
\int_{a}^{b} \mathcal{G} d x=(b-a) \mathcal{G}
$$

and,

$$
\begin{aligned}
\int_{a}^{b} \mathbf{P}(x) d x & =\left[\begin{array}{llll}
\int_{a}^{b} p_{0, n}(x) & \int_{a}^{b} p_{1, n}(x) & \ldots & \int_{a}^{b} p_{n, n}(x)
\end{array}\right] \\
& =\frac{b-a}{n+1}\left[\begin{array}{llll}
1 & 1 & \ldots & 1
\end{array}\right]
\end{aligned}
$$

from Theorem 1.1.

Substituting these relations into equation (2.3), the equation becomes

$$
\left(\frac{b-a}{n+1}\right) \sum_{k=0}^{m} \mathcal{A}_{k}\left[\begin{array}{llll}
1 & 1 & \ldots & 1
\end{array}\right] \mathbf{N}^{k} \mathcal{Y}=(b-a) \mathcal{G}
$$

Denoting the matrix $\mathcal{I}=\frac{1}{n+1}\left[\begin{array}{lllll}1 & 1 & 1 & \ldots & 1\end{array}\right]$, then the desired matrix relation is obtained as

$$
\sum_{k=0}^{m} \mathcal{A}_{k} \mathcal{I} \mathbf{N}^{k} \mathcal{Y}=\mathcal{G}
$$


Moreover, equation (2.4) and mixed conditions (1.2) can be written simply as follows:

$$
\begin{aligned}
\mathcal{W Y} & =\mathcal{G} \text { or }[\mathcal{W} ; \mathcal{G}] \\
\mathbf{M}_{i} \mathbf{Y} & =\beta_{i} \text { or }\left[\mathbf{M}_{i} ; \beta_{i}\right] ; i=0, \ldots, m-1
\end{aligned}
$$

so that $\mathcal{W}=\sum_{k=0}^{m} \mathcal{A}_{k} \mathcal{I} \mathbf{N}^{k}$ and $\mathbf{M}_{i}=\sum_{k=0}^{m-1} \sum_{j=0}^{l} \alpha_{i j}^{k} \mathbf{P}\left(c_{j}\right) \mathbf{N}^{k}$. Here matrix equation (2.5) corresponds to a linear algebraic system. Since $\mathcal{W}$ is a square matrix, this linear algebraic system can be solved easily. So, the values of unknown matrix $\mathcal{Y}$ are approximately found at the points $a+\frac{(b-a) i}{n}$.

\section{Numerical Results}

Linear differential equations with both constant and variable coefficients given respectively in the initial, boundary and mixed conditions are considered in numerical examples. The numerical results obtained by the Bernstein operator method are calculated by using adding and deleting techniques mentioned in [19] on the program MATLAB 7.1. In the tables, computations of the maximum errors and a comparison of the approximate solutions and exact solutions for the proposed method are given. So then, the using formulas can be defined as follows:

$$
\left|e_{n}(x)\right|=\left|y(x)-y_{n}(x)\right|, E_{\max }=\max _{x \in[a, b]}\left|e_{n}(x)\right|
$$

so that $y$ is exact solution and $y_{n}$ is the Bernstein operator approximate solution of the problem. Here attention should be paid that the numerical results are computed at the $x_{i}=a+\frac{(b-a) i}{n}$ points of function taken part in the Bernstein operator, instead of collocation points.

Example 3.1. Consider the following eighth order differential equation under the initial conditions [16]

$$
\begin{aligned}
y^{(8)}-y & =-8 e^{x} ; 0<x<1 \\
y(0) & =1, y^{\prime}(0)=0, y^{(2)}(0)=-2, y^{(3)}(0)=-2, y^{(4)}(0)=-3 \\
y^{(5)}(0) & =-4, y^{(6)}(0)=-5, y^{(7)}(0)=-6
\end{aligned}
$$

that has exact solution $y(x)=(1-x) e^{x}$.

Table 1. $E_{\max }$ errors of Example 3.1.

\begin{tabular}{|l|l|l|l|l|l|}
\hline$n$ & Adding tech. & \multicolumn{4}{|c|}{ Deleting tech. } \\
\hline & $\begin{array}{l}\text { adding without } \\
\text { deletion }\end{array}$ & $\begin{array}{l}\text { deleting last } \\
\text { rows }\end{array}$ & $\begin{array}{l}\text { deleting first } \\
\text { rows }\end{array}$ & $\begin{array}{l}\text { deleting first } \\
\text { and last rows }\end{array}$ & $\begin{array}{l}\text { deleting mid- } \\
\text { dle rows }\end{array}$ \\
\hline 10 & $8.7 e-004$ & $8.5 e-007$ & $5.5 e-005$ & $3.3 e-006$ & $6.0 e-006$ \\
\hline 15 & $7.2 e-007$ & $7.2 e-007$ & $5.5 e-007$ & $7.2 e-007$ & $7.2 e-007$ \\
\hline 20 & $5.4 e-007$ & $5.1 e-007$ & $2.3 e-004$ & $5.3 e-007$ & $9.2 e-006$ \\
\hline 25 & $4.4 e-007$ & $2.6 e-005$ & $6.1 e-001$ & $4.1 e-007$ & $3.6 e-004$ \\
\hline 30 & $4.5 e-007$ & $6.3 e-004$ & $4.9 e-001$ & $4.4 e-007$ & $3.2 e-001$ \\
\hline 35 & $2.6 e-007$ & $2.3 e-002$ & $9.6 e-001$ & $1.0 e-006$ & $5.4 e-001$ \\
\hline
\end{tabular}

In Table 1, the maximum errors of the Bernstein operator method are given. These results are calculated on the points $x_{i}=\frac{i}{n} ; n=0,1, \ldots n$. It shows that the values of the error shrinks slowly for increasing values $n$. Moreover, the numerical results calculated with deleting first and last rows are better then the others. For $n=35$, the best value is obtained by considering the adding technique.

Example 3.2. Consider the following fourth order differential equation under the boundary conditions [12] that have an exact solution $y(x)=e^{x}$ :

$$
\begin{gathered}
y^{(4)}-3 y=-2 e^{x} ; x \in[0,1] \\
y(0)=1, \quad y(1)=e, y^{\prime}(0)=1, \quad y^{\prime}(1)=e .
\end{gathered}
$$


Table 2. A comparison of the $y$ and $y_{n}$ solutions for Example 3.2.

\begin{tabular}{|c|c|c|c|c|c|}
\hline & $y(x)$ & \multicolumn{4}{|c|}{$y_{n}(x)$} \\
\hline$x$ & & $n=6$ & $n=8$ & $n=10$ & $n=12$ \\
\hline 0.1 & 1.1051709 & 1.1051698 & 1.1051575 & 1.1051598 & 1.1051617 \\
\hline 0.2 & 1.2214028 & 1.2213906 & 1.2213586 & 1.2213665 & 1.2213725 \\
\hline 0.3 & 1.3498588 & 1.3498251 & 1.3497802 & 1.3497945 & 1.3498052 \\
\hline 0.4 & 1.4918247 & 1.4917660 & 1.4917191 & 1.4917386 & 1.4917530 \\
\hline 0.5 & 1.6487213 & 1.6486438 & 1.6486042 & 1.6486262 & 1.6486420 \\
\hline 0.6 & 1.8221188 & 1.8220368 & 1.8220094 & 1.8220301 & 1.8220449 \\
\hline 0.7 & 2.0137527 & 2.0136831 & 2.0136683 & 2.0136844 & 2.0136958 \\
\hline 0.8 & 2.2255409 & 2.2254973 & 2.2254919 & 2.2255013 & 2.2255079 \\
\hline 0.9 & 2.4596031 & 2.4595885 & 2.4595877 & 2.4595907 & 2.4595928 \\
\hline
\end{tabular}

In Table 2, the Bernstein approximate solution and exact solution of the problem are compared for the different points and values $n$. The numerical results are calculated by considering the adding technique. The table shows that convergence of the Bernstein operator method are better for $x=0.1,0.2$ and $n=6$. For increasing $x$ and $n$ values, numerical results get worse.

Example 3.3. Let the following boundary value problem [32] be considered:

$$
\begin{gathered}
y^{(6)}+y=6(2 x \cos (x)+5 \sin x) ; x \in[-1,1] \\
y(-1)=y(1)=0, \quad y^{\prime}(-1)=y^{\prime}(1)=2 \sin (1), y^{\prime \prime}(-1)=-y^{\prime \prime}(1)=-4 \cos (1)-2 \sin (1)
\end{gathered}
$$

An exact solution of the problem is $y(x)=\left(x^{2}-1\right) \sin (x)$.

Table 3. $E_{\max }$ errors of Example 3.3.

\begin{tabular}{|c|l|l|l|}
\hline & Adding tech. & \multicolumn{2}{|c|}{ Deleting tech. } \\
\hline$n$ & $\begin{array}{l}\text { adding without } \\
\text { deletion }\end{array}$ & $\begin{array}{l}\text { deleting first } \\
\text { and last rows }\end{array}$ & $\begin{array}{l}\text { deleting mid- } \\
\text { dle rows }\end{array}$ \\
\hline 9 & $2.5 e-002$ & $7.4 e-004$ & $1.2 e-004$ \\
\hline 15 & $6.8 e-005$ & $6.8 e-005$ & $6.8 e-005$ \\
\hline 20 & $5.2 e-005$ & $5.2 e-005$ & $5.2 e-005$ \\
\hline 25 & $4.2 e-005$ & $4.2 e-005$ & $4.2 e-005$ \\
\hline 32 & $3.3 e-005$ & $4.3 e-005$ & $3.3 e-005$ \\
\hline 40 & $2.7 e-005$ & $2.4 e-002$ & $2.7 e-005$ \\
\hline 50 & $2.1 e-005$ & $4.2 e-001$ & $2.1 e-005$ \\
\hline 64 & $1.7 e-005$ & $3.5 e-001$ & $1.7 e-005$ \\
\hline 128 & $8.5 e-006$ & $3.7 e-001$ & $8.6 e-006$ \\
\hline
\end{tabular}

The maximum errors of the proposed method are given on the points $x_{i}=-1+\frac{2 i}{n} ; n=0,1, \ldots n$ in Table 3 . The table shows that the numerical results obtained by deleting technique better than the adding technique for $n=9$. Then, it is seen that the numerical results obtained by both adding and deleting techniques are same for $n=15,20,25$. The best numerical result is obtained by adding technique and for $n=128$.

Example 3.4. Consider the linear differential equation under the mixed conditions [15]:

$$
\begin{aligned}
y^{\prime \prime}+\log \sqrt[8]{(x+3)} y & =3 x \cos \left(x^{2}-2\right) \\
y(-1)-3 y^{\prime}(-1) & =0,2 y(1)-4 y^{\prime}(1)=0 .
\end{aligned}
$$

Table 4. Comparison of approximate solutions for Example 3.4.

\begin{tabular}{|c|c|c|c|c|c|c|}
\hline & \multicolumn{2}{|c|}{ Bernstein Operator Method } & \multicolumn{2}{c|}{ Nyström Method } & \multicolumn{2}{c|}{ Bernstein Collocation Method } \\
\hline$n$ & $x=-0.4$ & $x=0.7$ & $x=-0.4$ & $x=0.7$ & $x=-0.4$ & $x=0.7$ \\
\hline 8 & 0.343335 & 0.252831 & 0.222949 & 0.121655 & 0.220505 & 0.120426 \\
\hline 16 & 0.260116 & 0.208530 & 0.222945 & 0.121655 & 0.222949 & 0.121655 \\
\hline
\end{tabular}


The approximate solutions obtained by the Bernstein operator method and the other methods are given for different two points in Table 4. The numerical results of the proposed method are also calculated with the adding technique. By the table, the results of the Bernstein operator method have been compared with the results of the Nyström method [15] and the Bernstein collocation method [2]. However exact solution of the problem is not known, the table shows that the numerical results are decreased for increasing $n$ values.

\section{Conclusions}

In this paper, a new numerical method is generated by considering the generalized Bernstein polynomials as a sequence of the linear positive operators for finding solutions of the linear differential equations under the mixed conditions on the $C[a, b]$. The mentioned method has a lot of remarkable advantages. One of these, this method doesn't need to use the collocation points for approximating to unknown function of the differential equations. Because, the approximate solutions of the problems are found on the only points defined the generalized Bernstein polynomials by means of new method. This main point supports the Theorem 1.1 [2]. By considering this perpective, to approximate all the functions falled in the differential equations via the Bernstein polynomials is created appearance of the numerical method worked on. Alternatively, if the Theorem 2.1 is considered, operational matrix form of the Bernstein polynomials is enough to constitute this method. So, second of the advantages is obtained the theory of the proposed method simply. Third of these, this method is applied to calculate the numerical errors in the setting of the programme easily. The adding and deleting techniques used for computations of the numerical results are also compared in the Tables $[1,3]$. Looking to these tables, we can say that adding technique is more better than the deleting techniques for calculating the numerical results. In this reason, to use the adding technique is an effective way for computing numerical results. Besides, the numerical results of the method are not better more and more $n$ values from the tables [1-3]. In this case, to calculate the numerical results for smaller $n$ values is sufficient. In line with this developments, many numerical methods can be improved by considering the linear positive operators like Stancu-Chlodowsky operator [9] generalized Bernstein-Chlodowsky operator defined on the interval $[0, \infty)$ instead of the Bernstein operator. New methods derivated with this way can be transferred for approximate solutions of many types of differential, integral, integro-differential, fractional equations and other recallable equations in the future works.

\section{References}

[1] Akyuz-Dascioglu, A., Isler, N.: Bernstein Collocation Method for Solving Nonlinear Differential Equations. Mathematical and Computational Applications. 18 (3), 293-300 (2013).

[2] Akyüz-Daşcioğlu, A., Acar İşler, N.: Bernstein Collocation Method for Solving Linear Differential Equations. Gazi University Journal of Science. 26 (4), 527-534 (2013).

[3] Bataineh, A., Isik, O., Aloushoush, N., Shawagfeh, N.: Bernstein Operational Matrix with Error Analysis for Solving High Order Delay Differential Equations. Int. J. Appl. Comput. Math. 3, 1749-1762 (2017).

[4] Bhatta, D.D., Bhatti, M.I.: Numerical Solution of KdV equation using modified Bernstein polynomials. Applied Mathematics and Computation. 174 (2), 1255-1268 (2006).

[5] Bhattacharya, S., Mandal, B.N.: Use of Bernstein polynomials in numerical solutions of Volterra integral equations. Applied Mathematical Sciences. 2, 1773-1787 (2008).

[6] Bhattacharya, S., Mandal, B.N.: Numerical solution of a singular integro-differential equation. Applied Mathematics and Computation. 195, 346-350 (2008).

[7] Bhatti, M.I., Bracken, P.: Solutions of differential equations in a Bernstein polynomial basis. Journal of Computational and Applied Mathematics. 205 (2), 172-280 (2007).

[8] Bernstein, S.: Démonstration du théorème de Weierstrass Fondeé sur le calcul des probabilités . Commun. Soc. Math. Kharkow. 13 (2), 1-2 (1912).

[9] Büyükyazıc1, İ.: Approximation by Stancu-Chlodowsky polynomials. Computers and Mathematics with Applications. 59 (1), 274-282 (2010). 
[10] Doha, E.H., Bhrawy, A.H., Saker, M.A.: On the derivatives of Bernstein polynomials: An application for the solution of high even-order differential equations. Boundary Value Problems. 2011, 1-16 (2011).

[11] Doha, E.H., Bhrawy, A.H., Saker, M.A.: Integrals of Bernstein polynomials: An application for the solution of high even-order differential equations. Applied Mathematics Letters. 24, 559-565 (2011).

[12] El-Gamel, M.: A comparison between the Sinc-Galerkin and the modified decomposition methods for solving two-point boundary-value problems. Journal of Computational Pysics. 223 (1), 369-383 (2007).

[13] Faheem, K., Ghulam, M., Muhammad, O., Haziqa, K.: Numerical approach based on Bernstein polynomials for solving mixed Volterra-Fredholm integral equations. AIP Advances. 7, 1-14 (2017). https://doi.org/10.1063/1.5008818

[14] Farouki, R.T., Rajan, V.T.: Algorithms for polynomials in Bernstein form. Computer Aided Geometric Design. 5, 1-26 (1988).

[15] Frammartino, C.: A Nyström method for solving a boundary value problem on [-1, 1]. Calcolo. 47, 1-19 (2010).

[16] Golbabai, A., Javidi, M.: Application of homotopy perturbation method for solving eighth-order boundary value problems. Applied Mathematics and Computation. 191 (2), 334-346 (2007).

[17] Hesameddini, E., Khorramizadeh, M., Shahbazi, M.: Bernstein polynomials method for solving Volterra-Fredholm integral equations. Bull. Math. Soc. Sci. Math. Roumanie Tome 60. 108 (1), 59-68 (2017).

[18] Işık, O.R., Sezer, M., Güney, Z.: A Rational approximation based on Bernstein polynomials for high order initial and boundary value problems. Applied Mathematics and Computation. 217 (22), 9438-9450 (2011).

[19] İşler Acar, N., Daşcioğlu, A.: A projection method for linear Fredholm-Volterra integro-differential equations. Journal of Taibah University for Science. 13 (1), 644-650 (2019).

[20] Jani, M., Babolian, E., Javadi, S.: Bernstein modal basis: Application to the spectral Petrov-Galerkin method for fractional partial differential equations. Math Meth Appl Sci. 40, 7663-7672 (2017).

[21] Javadi, S., Babolian, E., Tahari, Z.: Solving generalized pantograph equations by shifted orthonormal Bernstein polynomials. Journal of Computational and Applied Mathematics. 303, 1-14 (2016).

[22] Kadkhoda, N.: A numerical approach for solving variable order differential equations using Bernstein polynomials. Alexandria Engineering Journal. (2020). https:/ / doi.org/10.1016/j.aej.2020.05.009

[23] Korovkin, P.P.: On convergence of linear positive operators in the space of continuous functions. Dokl. Akad. Nauk SSSR (N.S). 90, 961-964 (1953).

[24] Lorentz, G.G.: Bernstein polynomials. Chelsea Publishing. New York (1986).

[25] Mirzaee, F., Samadyar, N.: Parameters estimation of HIV infection model of CD4+ T-cells by applying orthonormal Bernstein collocation method. International Journal of Biomathematics. 11 (2), 1-19 (2018).

[26] Mohamadi, M., Babolian, E., Yousefi, S.A.: A Solution For Volterra Integral Equations of the First Kind Based on Bernstein Polynomials. Int. J. Industrial Mathematics. 10 (1), 1-9 (2018).

[27] Ordokhani, Y., Davaei far, S.: Approximate solutions of differential equations by using the Bernstein polynomials. International Scholarly Research Network ISRN Applied Mathematics. 2011 (1), 1-15 (2011).

[28] Parand, K., Sayyed, A., Hossayni, J.A.R.: Operation matrix method based on Bernstein polynomials for the Riccati differential equation and Volterra population model. Applied Mathematical Modelling. 40, 993-1011 (2016).

[29] Pirabaharan P., Chandrakumar, R.D.: A computational method for solving a class of singular boundary value problems arising in science and engineering. Egyptian journal of basic and applied sciences. 3, 383-391 (2016).

[30] Quasim, A.F., Hamed, A.A.: Treating Transcendental Functions in Partial Differential Equations Using the Variational Iteration Method with Bernstein Polynomials. Hindawi International Journal of Mathematics and Mathematical Sciences. 2019, 1-8 (2019). https:/ / doi.org/10.1155/2019/2872867 
[31] Quasim, A.F., Al-Ravi, E.S.: Adomian Decomposition Method with Modified Bernstein Polynomials for Solving Ordinary and Partial Differential Equations. Hindawi Journal of Applied Mathematics. 2018, 1-9 (2018). https://doi.org/10.1155/2018/1803107

[32] Ramadan, M.A., Lashien, I.F., Zahra, W.K.: High order accuracy nonpolynomial spline solutions for 2 th order two point boundary value problems. Applied Mathematics and Computation. 204 (2), 920-927 (2008).

[33] Rani, D., Mishra, V.: Approximate Solution of Boundary Value Problem with Bernstein Polynomial Laplace Decomposition Method. International Journal of Pure and Applied Mathematics. 114 (4), 823-833 (2017).

[34] Saadatmandi, A.: Bernstein operational matrix of fractional derivatives and its applications. Applied Mathematical Modelling. 38, 1365-1372 (2014).

[35] Shirin, A., Islam, A.S.: Numerical solutions of Fredholm integral equations using Bernstein polynomials. Journal of Scientific Research. 2 (2), 264-272 (2010).

[36] Siddiqi, S.S., Akram, G.: Septic spline solutions of sixth-order boundary value problems. Journal of Computational and Applied Mathematics. 215 (1), 288-301 (2008).

[37] Şuayip, Y.: A collocation method based on Bernstein polynomials to solve nonlinear Fredholm-Volterra integro-differential equations. Applied Mathematics and Computation. 273, 142-154 (2016).

[38] Yi-ming, C., Li-qing, L., Dayan, L., Driss, B.: Numerical study of a class of variable order nonlinear fractional differential equation in terms of Bernstein polynomials. Ain Shams Engineering Journal. 9, 1235-1241 (2018).

[39] Yousefi, S.A., Dehghan, B.M.: Bernstein Ritz-Galerkin method for solving an initial-boundary value problem that combines Neumann and integral condition for the wave equation. Numerical Methods for Partial Differantial Equations. 26, 1236-1246 (2009).

\section{Affiliations}

NEŞE İşLER ACAR

AdDRESS: Mehmet Akif Ersoy University, Department of Mathematics, 15030, Burdur, Turkey.

E-MAIL: nisler@mehmetakif.edu.tr

ORCID ID: 0000-0003-3894-5950 